\title{
Classification of the primary progressive aphasias: principles and review of progress since 2011
}

\author{
Rik Vandenberghe $e^{1,2,3}$
}

\begin{abstract}
Highly influential recommendations published in 2011 for the classification of the primary progressive aphasias (PPA) distinguished three subtypes: the semantic variant, the nonfluent/agrammatic variant, and the logopenic variant. We review empirical evidence published after 2011 that bears relevance to the validity of the recommended classification scheme. The studies that we review principally rely on monocentric, memory clinic-based consecutive series of PPA patients. We review whether a data-driven analysis of neurolinguistic test scores confirms the subtyping that was based on expert consensus, whether the 2011 subtyping covers the diversity of PPA in a comprehensive manner, and whether the proposed subgroups differ along dimensions that are not explicitly part of the defining criteria, such as diffusion tractography. Data-driven mathematical analyses of neurolinguistic data in PPA broadly confirm the presence of separate clusters corresponding to the subtypes but also leave 15-30\% unclassified. A comprehensive description of PPA requires the addition of the mixed variant as a fourth subtype and needs to leave room for cases fulfilling the criteria for a root diagnosis of PPA but not those of any of the three subtypes. Finally, given the limited predictive value of the clinical phenotype for the underlying neuropathology, biomarkers of the underlying pathology are likely of clinical utility in PPA.
\end{abstract}

\section{Background}

Consensus recommendations for the classification of primary progressive aphasia were published in 2011 [1], partly motivated by the need to consolidate the logopenic variant (LV) $[2,3]$ as a third subtype in addition to the nonfluent/agrammatic variant (NFV) [4] and the semantic variant (SV). The latter is also known as semantic dementia [5]. The LV is associated with substantially higher probability of Alzheimer's disease (AD) compared with the other two subtypes-hence its clinical relevance $[2,3]$. We describe the principles of the PPA classification scheme and review evidence that appeared after the recommendations were published and that bears on the validity of this classification scheme and may also point to possible ways in which the classification scheme could be further improved.

Correspondence: rik.vandenberghe@uz.kuleuven.ac.be

${ }^{1}$ Department of Neurosciences, Laboratory for Cognitive Neurology, KU Leuven, Leuven, Belgium

${ }^{2}$ Neurology Department, University Hospitals Leuven, Leuven, Belgium Full list of author information is available at the end of the article

\section{The current recommendations for classifying PPA cases \\ Root diagnosis of PPA}

The root diagnosis of PPA is based on the objective impairment of language while other cognitive domains (episodic and topographical memory, constructional praxis, etc.) are relatively preserved [6]. In the initial disease stages, impacts on the instrumental activities of daily living are entirely attributable to language problems.

Confusion may arise when the root diagnostic criterion is not fulfilled and the subtyping is applied nevertheless. In that respect it is of particular importance not to confuse PPA or any of its subtypes with the left hemisphere-dominant type of clinically probable $\mathrm{AD}$ with prominent language symptoms, a disease entity that has been well-known for a long time [7, 8]. In PPA due to $\mathrm{AD}$, tests of nonverbal domains-for instance, copy of the overlapping pentagons in the Mini Mental State examination or ideomotor praxis-should be, by definition, preserved. On the other hand, in left hemispheredominant clinically probable $\mathrm{AD}$, some other cognitive 
domains besides language are, by definition, also affected and the PPA root criterion is therefore not fulfilled. Some clinical features may be particularly discriminative, such as tests for constructional praxis. Other tests, e.g., of verbal episodic memory, may be less reliable as they will be influenced by the language problems. Neither are nonverbal episodic memory tests particularly useful in this respect in our experience as encoding and retrieval can be affected by the executive dysfunction that can occur in PPA.

Of patients who fulfilled the root criterion of PPA, $40 \%$ had underlying AD [9]. When AD causes PPA, the neuropathology is atypical because of the asymmetric, left hemisphere-dominant distribution of tangles and because of the higher ratio of neocortical-to-entorhinal tangles [10].

\section{The logopenic variant}

In PPA LV, spontaneous speech is halting and characterized by fluency disruptions with incomplete words and hesitations [11]. Word finding pauses frequently occur after determiners preceding content words [12]. Grammatical processing and motor speech are relatively preserved (Fig. 1).

Testing repetition is key to the diagnosis of LV. It is critical not to rely on a global repetition score but to take into account the subscores for the different types of materials. In LV, the repetition deficit characteristically affects repetition of longer or complex sentences [13] rather than single polysyllabic words [13, 14]. This differs from NFV with speech apraxia where polysyllabic words will be affected by the motor speech deficit, in particular when consonant clusters are present. One of the least specific and most sensitive tests for repetition concerns the repetition of a string of function words, as in the MiniMental State Examination test. This item can be impaired across the entire spectrum of PPA as well as early in typical AD.

The cognitive deficit underlying the repetition deficit in LV is most likely a short-term phonological memory deficit [3]. In LV, the digit span forward is typically decreased and this deficit does not extend to short-term memory for tones or for location [15]. This clearly differs from SV where digit span forward is often preserved until late into the disease course.

In a hierarchical clustering analysis of 32 cases with PPA excluding semantic variant, cases could be grouped into four clusters [13]. Cluster 1 was characterized by anomia and deficient repetition of sentences and corresponded to LV PPA, cluster 2 to NFV. Cluster 1a was further characterized by phonological paraphasias while cluster $1 \mathrm{~b}$ lacked these. In cluster $1 \mathrm{a}$ all cases were amyloid-positive on positron emission tomography (PET) compared with three out of seven cases in cluster

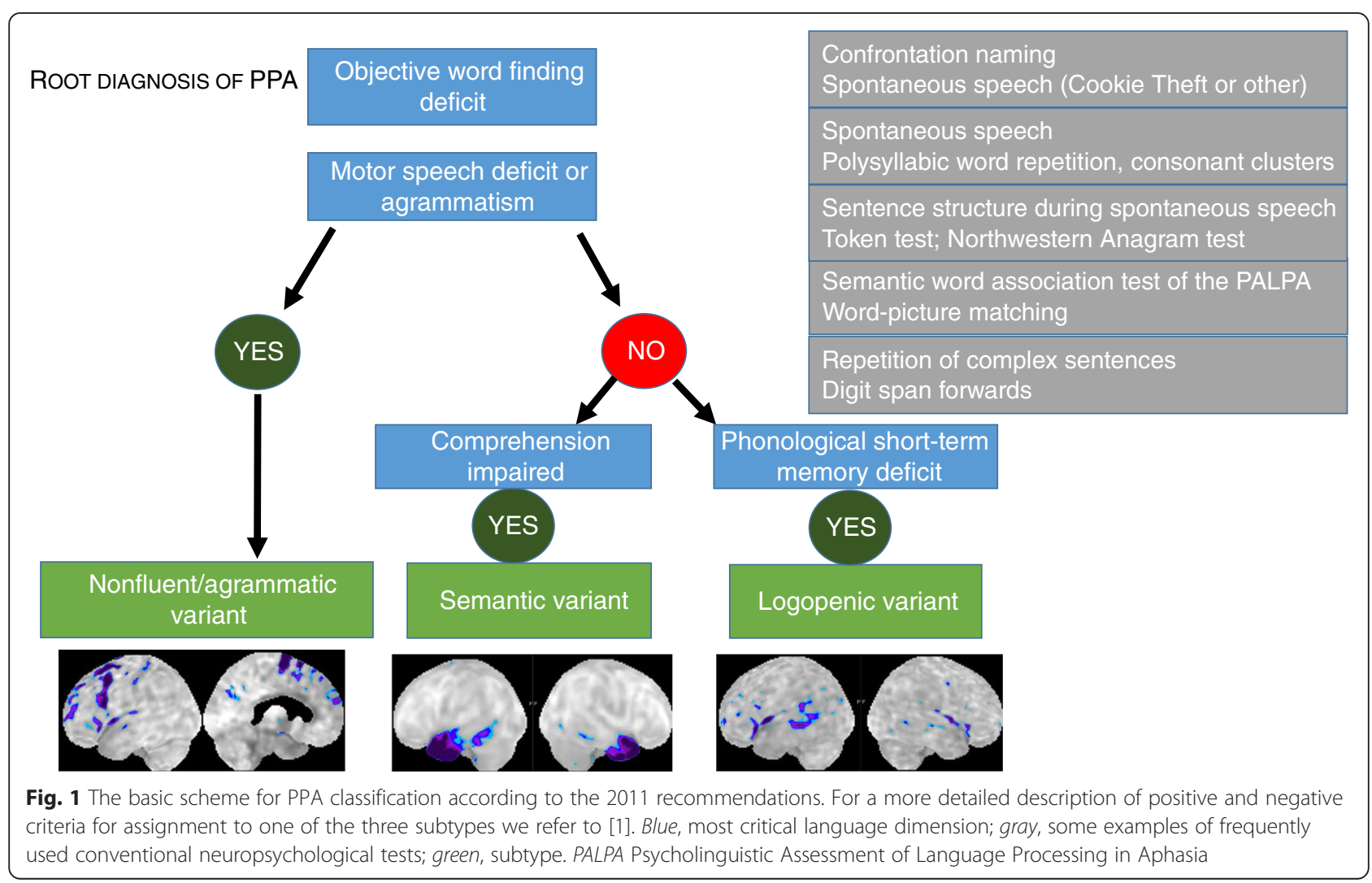


$1 b$ [13]. Across the entire group of non-SV PPA [13], the tests that best discriminated the amyloid-positive from the amyloid-negative cases were the presence of phonological paraphasias and the absence of agrammatism and motor speech disorder. Sentence comprehension and anomia as such did not have discriminative value within this non-SV PPA group [13].

This and other studies suggest that the LV encompasses at least two subtypes [12-14, 16]: one closely resembles left hemisphere-dominant probable $\mathrm{AD}$ and the other constitutes a more restricted clinical and anatomical phenotype more similar to the original description [2]. This led Teichmann et al. [12] to propose the term "logopenic aphasia complex".

In LV, as the disease progresses [16], language impairment may become more widespread [12], leading to problems with single word comprehension [16, 17], single word repetition, syntax production, and verbal memory [14]. Non-language cognitive domains may become affected [14] (for instance, constructional praxis) in such a way that the pattern blends with left hemispheredominant clinically probable AD [16]. This clinical course is distinct from that seen in SV where the steady progression typically remains restricted to language, semantic memory, and executive dysfunction [16]. Other patients with LV retain a very circumscribed deficit restricted for years to sentence repetition and word finding difficulties and focal atrophy.

In LV cluster 1a [13], the group-based pattern of atrophy, perfusion, and metabolism resembled that seen in the left hemisphere-dominant, clinically probable $\mathrm{AD}$ cases [12, 14, 18, 19] (Figs. 1 and 2a). In cluster 1b the damage was more restricted [13] to the temporoparietal transition zone, as originally described by Gorno-
Tempini et al. [2, 3] (Fig. 2b). This probably is the key region responsible for the repetition deficit in LV and belongs to the dorsal language pathway [20]. Atrophy in the temporoparietal transition zone correlates with repetition scores and with gesture imitation scores in PPA [21]. In LV, the white matter tracts underneath this cortical region, i.e., the temporoparietal portion of the superior longitudinal fascicle, are also damaged [22].

A second, nearby region that has been implicated in the phonological errors seen in LV is the posterior superior temporal cortex $[12,13]$. The posterior superior temporal sulcus is an area of predilection in typical $\mathrm{AD}$ from the preclinical [23] to the early clinical stage [24] and activity in this region correlates with word finding scores in clinically probable AD [24] as well as naming latency in preclinical AD [23]. Gray matter density in this region also correlates in LV with scores on lexical decision for words with a meaning that strongly relates to sounds (such as "thunder") [25].

The positive predictive value of the LV phenotype for a pathological diagnosis of $\mathrm{AD}$ is $50-60 \%[9,26,27]$, which is lower than what is found in in vivo amyloid biomarker studies in LV $(60-90 \%[12,28])$. In a metaanalysis of PPA LV series from different centers, the remaining $38 \%$ were due to Tar DNA binding protein 43 (TDP)-associated frontotemporal lobar degeneration (FTLD) [26], with 5-10\% caused by tau-associated FTLD [26]. LV can also be caused by AD combined with Lewy body pathology $[9,27]$.

\section{The semantic variant}

PPA SV is a distinct disease entity throughout the disease course clinically, anatomically, and neuropathologically-hence the frequently used name "semantic dementia" [5].

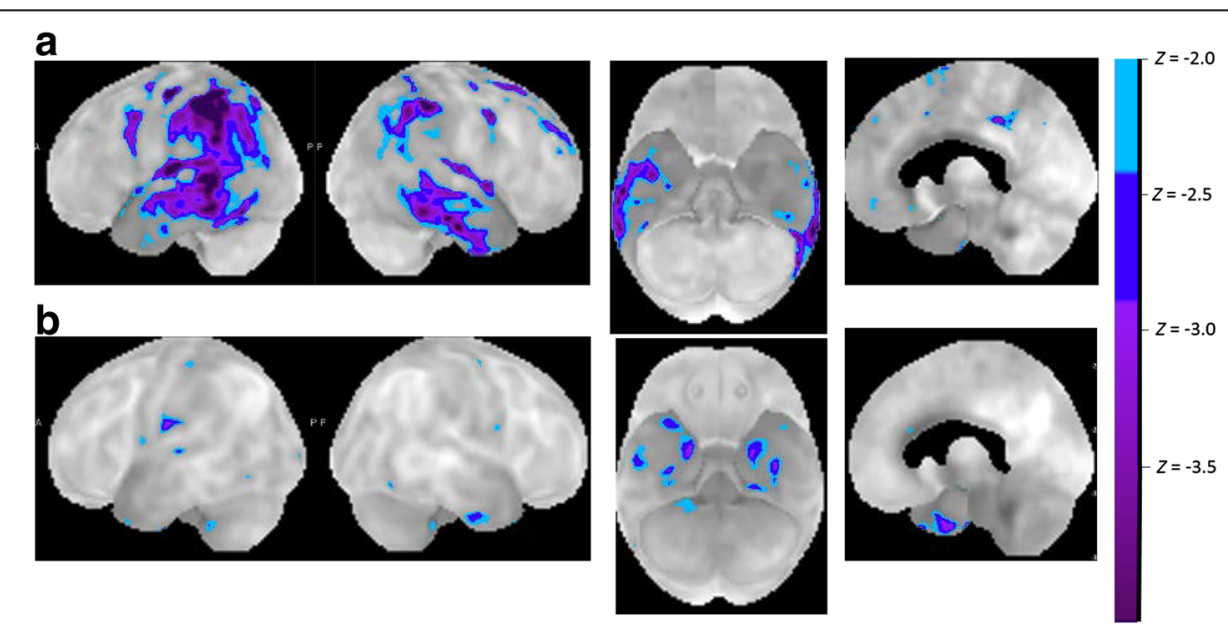

Fig. 2 Metabolic patterns in LV. a Patient with LV PPA and a pattern characteristic of left hemisphere-dominant Alzheimer's disease. This would correspond to cluster 1a. b Patient with LV PPA and a much more restricted pattern of hypometabolism. This would correspond to cluster $1 \mathrm{~b}$. The $Z$ maps are calculated by comparing the individual glucose metabolic pattern to the normal age-matched control database using commercial MIMVISTA software (MIM Software Inc., Cleveland, OH, USA), with a range between 50 and 80 years 
Characteristic features apart from anomia are word comprehension deficit and object recognition problems (Fig. 1). In $\mathrm{SV}$, spontaneous speech is fluent, with proportionally fewer nouns and open-content words than in any of the other subtypes and an increase in generic words [11]. As the disease progresses, spontaneous speech becomes restricted to stereotyped utterances consisting of a handful of connected words that may persist for several years and also echolalia. Even in an advanced stage, the clinical neurological examination often remains relatively intact.

Confrontation naming is often severely impaired, and more so than in LV at a comparable stage [6,9]. Naming errors occur initially, mainly for unfamiliar or atypical items [29], and consist of semantic paraphasias, generalizations, omissions, and circumlocutions. Patients may be able to comprehend the words that they cannot retrieve [30], but as the disease advances they may also not be able to comprehend the word. Object recognition problems and loss of knowledge of visual features of objects further contribute to the confrontation naming deficit in SV. In the written modality, loss of word meaning leads to surface dyslexia [31]. Patients may also experience problems identifying persons (knowledge about individuals) beyond proper name anomia.
The severity of comportmental and personality changes may vary in SV. Both anatomically and clinically, SV can overlap with frontotemporal dementia behavioral variant (FTD bv). The relative preponderance of aphasia versus comportmental disturbances depends on the direction and degree of the left-right asymmetry in the anterior temporal cortex [32].

During stages when word comprehension and object identification are still relatively preserved, SV can resemble LV, but in these circumstances the repetition deficit for specific types of materials, i.e., complex sentences with high working memory demands and the digit span forward, is a discriminative sign in favor of LV. If impaired repetition occurs in an early stage of SV, it is most often restricted to series of function words, a sensitive test for repetition deficit across all three types of PPA.

SV is characterized by a very distinct pattern of anterior temporal atrophy and hypometabolism (Figs. 1 and 3). This may be lateralized to the left or may also affect the right side. Different regions within the anterior temporal cortex may contribute different aspects to the clinical syndrome of SV. Perirhinal cortex belongs to the region of atrophy in semantic dementia [33-35] and scores on tests
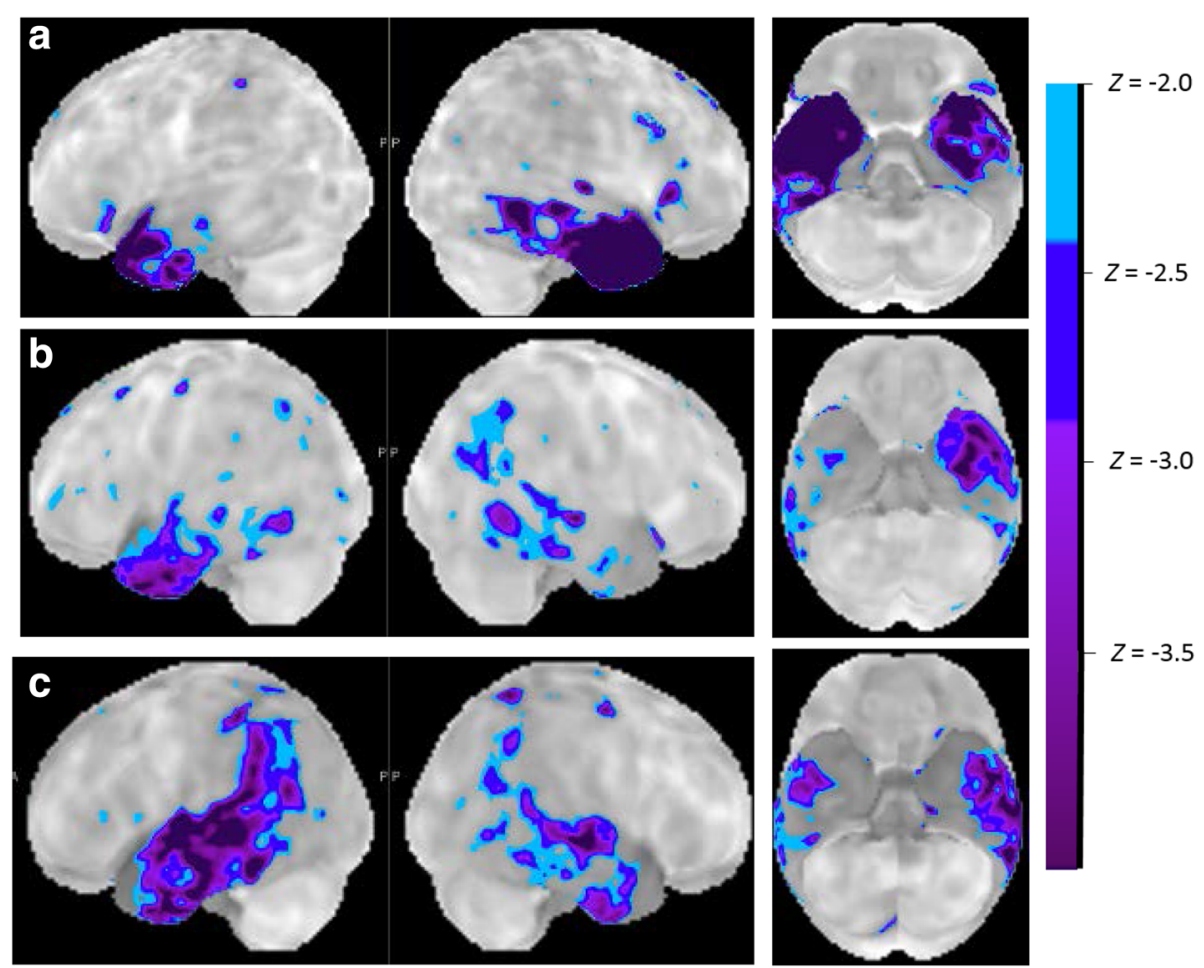

Fig. 3 Metabolic patterns. a Typical SV PET pattern. b, c Two PPA cases with a combination of left anterior temporal hypometabolism together with posterior inferior temporal and inferior parietal hypometabolism. This could pose diagnostic problems as there are features of both $A D$ and SV. In the right hemisphere, however, the hypometabolism is more pronounced in posterior than in anterior temporal cortex, suggesting that the damage progressed in the left hemisphere from posterior to anterior temporal cortex. In both cases cerebrospinal fluid AD biomarkers were clearly within the typical $A D$ range $(A \beta 42=393 \mathrm{pg} / \mathrm{ml}$ and total tau $=458 \mathrm{pg} / \mathrm{ml}$ in $\mathbf{b}$; $A \beta 42=525 \mathrm{pg} / \mathrm{ml}$ and total tau >1200 in c) 
of semantic memory as well as confrontation naming scores correlate with volume loss in temporopolar cortex and perirhinal cortex [33, 34]. In healthy individuals functional MRI activity patterns in perirhinal cortex, overlapping with the atrophic regions in SV, reflect the semantic similarities between written words [36]. In typical SV, the atrophy may often also impinge on anterior inferior frontal cortex and orbitofrontal cortex [19], which is connected with the anterior temporal pole through the uncinate fascicle. The uncinate fascicle is damaged in SV compared with LV, as well as the inferior longitudinal fascicle $[22,37]$. The latter may contribute to functional effects at a distance in the posterior fusiform cortex or the ventral occipitotemporal transition zone. This may account for the visuoperceptual identification problems (structural description system) and the loss of knowledge of visual features that are an integral part of the clinical picture in many SV patients, despite the fact that these regions as such are structurally relatively intact $[29,38]$.

In rare cases, the anterior temporal damage may be part of a more distributed atrophy and hypometabolism extending contiguously into more posterior temporal cortex and even inferior parietal cortex (Fig. 3b, c). Such a pattern then corresponds to the typical SV anterior temporal region plus the typical $\mathrm{AD}$ posterior temporal and inferior parietal region. Distinguishing between SV and left hemisphere-dominant clinically probable $\mathrm{AD}$ may require biomarker examination in these circumstances.

In a multicenter series, SV was associated with FTLDTDP in $69-83 \%$ of cases [26, 39], usually of type C [9]. AD has been described in up to $25 \%$ of PPA SV cases $[9,26]$, although in most series prevalence of AD in SV is substantially lower (10 \%) [40]. In SV due to AD, there is more entorhinal, hippocampal, parahippocampal, and temporal neocortical volume loss compared with nonAD SV [40]. FTLD-tau, principally Pick's disease, can also occur as a cause $[26,39]$.

\section{Nonfluent/agrammatic variant}

PPA NFV is characterized by agrammatism and/or speech apraxia (Fig. 1). Agrammatism refers to pathological changes in the morphology of nouns and verbs, word order, and argument structure, with a decrease in mean length of utterance and a decrease in sentence complexity [11]. For a detailed description of the diagnostic features of speech apraxia we refer to Josephs et al. [41, 42]. Schematically, speech apraxia is diagnosed based on abnormal duration of voxels and of intersegmental intervals (with segments referring to sounds, syllables, or words), unevenness in loudness and pitch and abnormalities in intonational stress, and sound distortions and substitutions, in particular for utterances of increased length and articulatory complexity [41]. This leads to phonetic errors. Phonetic errors must be distinguished from phonological errors which occur in LV in the context of normal prosody and normal sound production and from starting errors that are seen in LV. According to an automated analysis of the speech characteristics in NFV, the changes in relative duration and intensity of vowels as well as the pauses during reading are the most distinctive features [43]. Other qualitative features that may be helpful in the diagnosis of speech apraxia consist of sound and syllable repetitions, groping and effortful speech, speech initiation problems, and abnormalities in coordination with breathing [41].

Most often agrammatism and motor speech deficits occur together (9 out of 25 PPA in Mesulam et al. [6]). More rarely, the motor speech deficit may occur in isolation (1 out of 25 in Mesulam et al. [6]). The latter phenotype has been termed "progressive apraxia of speech" [41]. Since the deficit is limited to motor speech and does not affect language processing strictly speaking, Josephs et al. [41] have argued that this should be set apart from PPA. Inversely, agrammatism can also occur without speech apraxia: a hierarchical clustering analysis in 32 non-SV PPA patients revealed two clusters corresponding to NFV, one in which motor speech disorder co-occurred with agrammatism and one with agrammatism alone [13]. Phenotypically, therefore, one can distinguish three further subtypes within NFV PPA, depending on whether the agrammatism and speech apraxia occur in isolation or together. Over time the exclusivity may disappear [44].

A conspicuous source of heterogeneity within NFV are the associated neurological signs and symptoms that may be present at the initial clinical examination or may appear over the disease course [44]. These signs may point to progressive supranuclear palsy (PSP) or corticobasal degeneration (CBD) as the underlying cause. In 13 patients with progressive apraxia of speech, five evolved into a PSP-like syndrome while in the remaining subjects the speech problems continued to be the most prominent symptom along with progressive extrapyramidal signs [44].

In progressive apraxia of speech, FDG-PET mainly reveals hypometabolism in superior premotor and supplementary motor areas [44, 45] (Fig. 4). Superior premotor involvement correlates with the degree of speech apraxia [45]. As the disease progresses, regions at a distance may become involved, such as inferior parietal or posterior temporal cortex [19]. In the agrammatic variant a more distributed network is involved, including pars orbitalis, triangularis, and opercularis along with superior temporal gyrus and inferior parietal lobule $[6,45]$. Therefore, speech apraxia and agrammatism rely on anatomically dissociable mechanisms. Likewise, the white matter tracts involved differ depending on the degree of speech apraxia versus agrammatism [22]: speech 


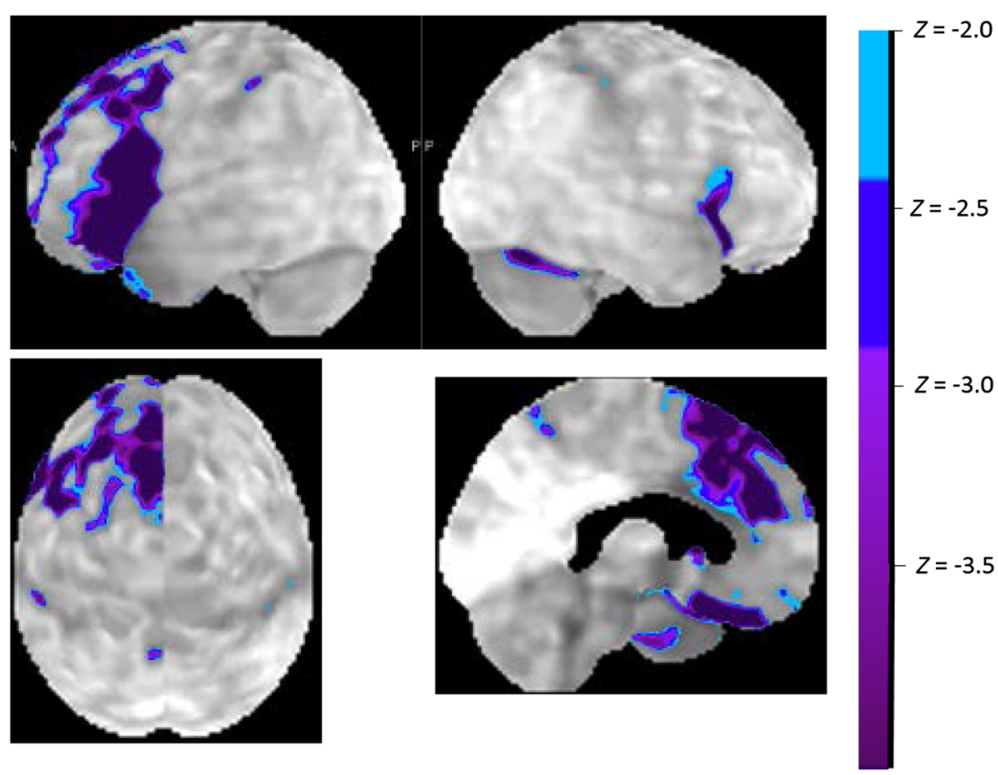

Fig. 4 Typical metabolic pattern in NFV showing hypometabolism in inferior frontal, premotor, and supplementary motor cortex

production scores mainly correlate with the white matter tract from the inferior frontal gyrus (Brodmann area (BA) 44) and the anterior insula to premotor (BA6 face/ mouth area) and supplementary motor cortex (the Aslant tract [46]) as well as connections with putamen and caudate [47]. Sentence comprehension/production, on the other hand, correlates with involvement of the superior longitudinal fascicle and the arcuate fascicle $[11,47]$. The dissociation of white matter tract involvement between speech apraxia and agrammatism is not absolute: motor speech scores also show some correlation with integrity of SLF and arcuate [48].

In NFV, $50-70 \%$ of patients are neuropathologically diagnosed as FTLD-tau, corresponding to corticobasal degeneration, progressive supranuclear palsy or Pick's disease $[9,26,39]$. Exclusive or predominant apraxia of speech predicts a tauopathy rather than a TDP43 proteinopathy [27] and may be more frequently associated with PSP than with CBD [39]. Approximately $20 \%$ of NFV cases are due to FTLD-TDP, usually of type A [27], and $12-25 \%$ to $\mathrm{AD}[9,26]$.

\section{Cases who do not fit into any of the three classes}

In a monocentric series of 84 PPA patients [49], $31 \%$ could not be assigned to any of the three subtypes. In another monocentric retrospective series, this occurred for 5 out of 30 PPA cases [27]. In a third monocentric longitudinal PPA series of 46 patients, $41 \%$ did not fulfill criteria of any of the three subtypes according to Sajjadi et al. [50]. In the latter series the proportion of LV was only $4 \%$ [50], suggesting that the unclassifiables contained cases that in other series could have been assigned to LV. Indeed, in a series by Sajjadi et al. [51] volumetric analysis of 14 unclassified patients revealed an "LV PPA like" pattern. This led the authors to conclude that the unclassifiable cases are more likely "AD-related aphasias." A case can be unclassifiable because it is missing some of the positive features that are necessary for assigning it to one of the three subtypes proposed by Gorno-Tempini et al. [1]. A case can also be unclassifiable because positive features belonging each to different subtypes occur in combination. The mixed variant is the most typical example of the latter.

\section{The mixed subtype}

The mixed subtype exhibits word comprehension deficits along with speech apraxia or agrammatism, a combination of positive findings that does not occur in the current classification [6,52] (Fig. 5). In our experience, this mixed variant is not rare. In a consecutive series of PPA cases at our memory clinic, 3 out of 21 are of the mixed variant, a proportion similar to that reported by Mesulam et al. [6] (2 out of 25). A group-based volumetric analysis of mixed PPA revealed atrophy in inferior frontal, superior temporal, and anterior temporal cortex [6]. Among six patients with mixed variant PPA, four had $\mathrm{AD}$ as underlying pathology, one had FTLD-tau, and one had mixed pathology of AD and TDP-A [9].

\section{Anomic-only PPA}

Patients may fulfill the root criterion of PPA based on their anomia, in the absence of any of the other features that distinguish between subtypes, most notably normal performance on repetition of complex sentences and on 


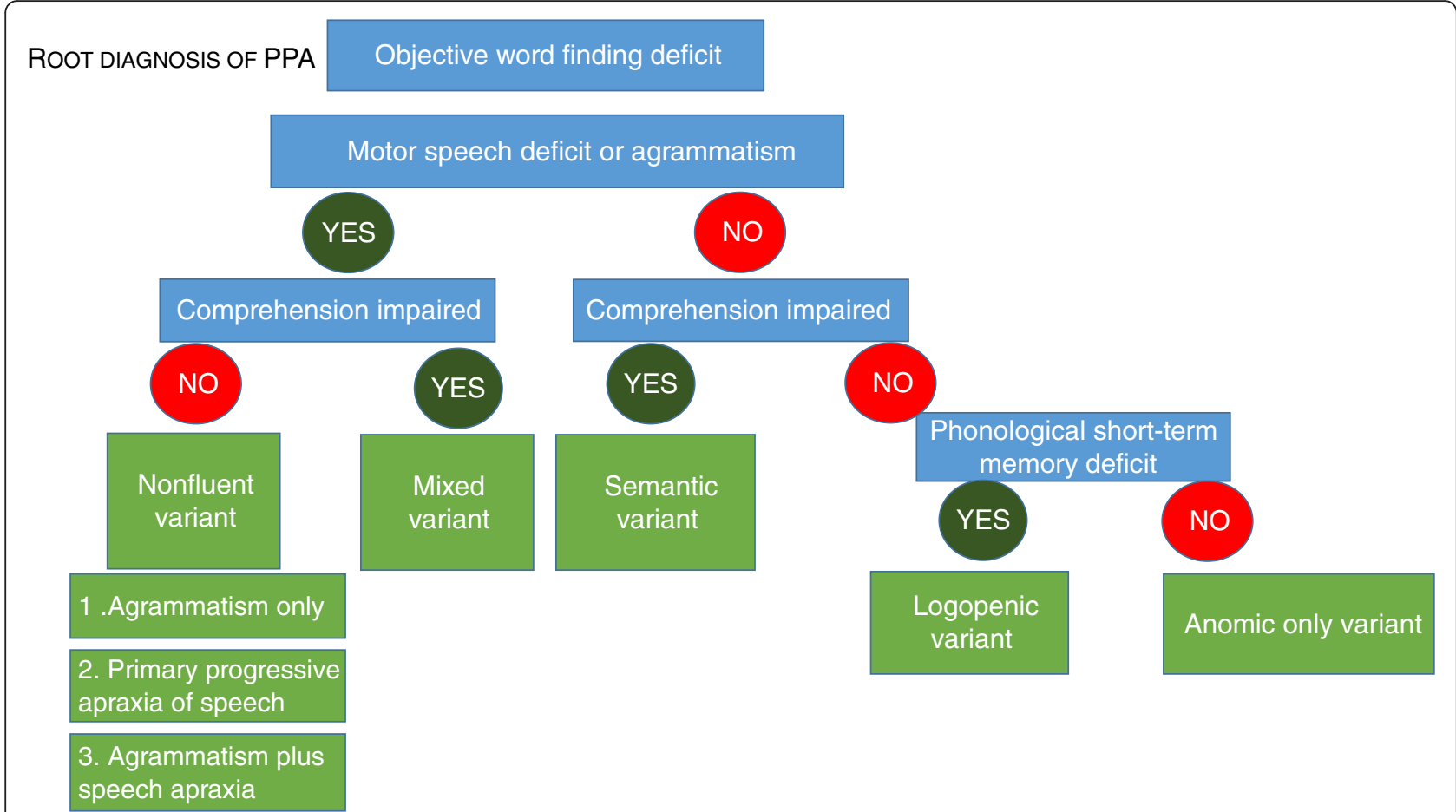

Fig. 5 Based on the literature reviewed after the 2011 recommendations, some modifications may be worth considering when describing cohorts for research. Currently the clinical impact of these modifications is insufficiently documented to recommend this updated scheme for use in clinical practice

word comprehension and no motor speech problems that would permit assignment to one of the three subtypes (Fig. 5). The prevalence in a series of PPA patients was 3 out of 25 [6]. A somewhat related subclass are PPA-L*, who have prominent word finding problems during spontaneous speech but have relatively preserved confrontation naming. PPA-L* have a hesitant spontaneous speech but are missing the repetition deficit typical of LV [9]. It is conceivable that these cases may develop some of the distinguishing features later in the course of the disease.

\section{Towards an etiological diagnosis of PPA}

The clinical utility of biomarkers such as cerebrospinal fluid A 342 and amyloid PET in patients with PPA presenting in a memory clinic depends on the difference between pre- and post-test odds of an etiological diagnosis [53] and on the clinical benefit of potential changes in patient management.

\section{Increase in diagnostic accuracy}

None of the three phenotypic subtypes entirely excludes the possibility of $\mathrm{AD}$ as the underlying cause. PPA LV has the highest positive predictive value for $\mathrm{AD}$ neuropathology but, even so, neuropathological series show a prevalence of AD pathology in PPA LV of only 50-60\% $[9,26]$. Inversely, PPA SV or NFV are far more often due to FTLD but can be caused by AD in $10-25 \%$ of cases $[9,26]$. The prevalence of $\mathrm{AD}$ as the underlying cause in the other categories, such as mixed or anomic variant, is currently unknown. Hence, the highest yield of biomarkers would probably be obtained in LV, anomic or mixed-variant PPA, and in SV in an early phase.

\section{Clinical utility}

The clinical utility of an etiological diagnosis in PPA has not been proven empirically. Conceivably, patients with PPA due to AD may have a cholinergic deficit and hence benefit from cholinesterase inhibitors. Prognostically, the time course expected in patients with PPA due to AD may differ from that in PPA due to a TDP43 proteinopathy or a tauopathy such as CBD or PSP. For instance, the behavioral manifestations may be qualitatively different in FTLD compared with $\mathrm{AD}$, with more obsessive-compulsive behaviors, lack of empathy, and eating abnormalities. In case of PPA due to tauopathy, limb apraxia, gait and postural problems, and dysphagia may impair the autonomy of the patient earlier in the course of the disease compared with PPA due to AD. Inversely, the cognitive domains affected over the disease course in $\mathrm{LV}$ due to $\mathrm{AD}$ may expand into nonverbal domains. A correct etiological diagnosis at the initial stage may help the patient, caregiver, and physician to anticipate specific problems which may benefit patient management. A correct etiological diagnosis in vivo could 
also be a prerequisite for success in clinical drug development aimed at disease modification.

\section{Need for modification of the criteria? Unclassified PPA}

In itself the observation that a substantial proportion of cases who fulfill the root criterion of PPA do not fall into one of the subtypes (the anomic-only variant) is not necessarily a reason for a revision, as long as these cases are not "forced" into one of the subtypes when communicating research findings or in clinical practice. It is important to recognize in the clinic and in research that the three subtypes do not encompass the full spectrum of PPA phenotypes, as described above [27]. The question then becomes whether within the unclassifiable cases some patterns can be discerned that merit the status of an additional subtype (Fig. 5).

\section{Addition of subtypes}

For the purpose of communicating research findings we believe that there is sufficient evidence and also a need to allow for one additional denotation: the mixed subtype. Based on the prevalence reported, ignoring this subtype would lead to misclassification of about $15 \%$ of cases in research papers on PPA. This additional subtype constitutes an important target group for further research to determine the anatomical and etiological basis and the potential relevance for patient treatment.

\section{Subdivision of subtypes}

Within the NFV, a more fine-grained level of description would specify the agrammatic-only variant, the speech apraxia-only variant, and the combination of both [42]. There are two caveats: it remains to be proven that the underlying neuropathology differs sufficiently between these subdivisions; and such a subdivision heavily relies on evidence that either motor speech or grammatical processing is preserved and this critically depends on the sensitivity of tests and the disease stage.

Within the LV it may be more accurate to distinguish between a subgroup with more widespread language involvement and broader anatomical involvement resembling left hemisphere-dominant $\mathrm{AD}$ and a subgroup with LV restricted to phonological working memory deficit and focal damage of the temporoparietal transition zone. The more restricted form has a lower likelihood of $\mathrm{AD}$ than the more widespread form.

\section{Conclusions}

Obviously, from the patient and caregiver perspective, a revision would be justified if it has a positive impact on the management of individual patients, as was the case, e.g., for the LV subtype as a recognition of $\mathrm{AD}$ as the frequent underlying cause. The 2011 recommendations had an impact beyond research. Before a modification would be deemed of use for clinical practice, more empirical data would be needed regarding the impact of these modifications on prediction of the temporal course or neuropathology and on patient management. Clinically, in our opinion, a higher benefit will be gained from implementing in vivo biomarkers of underlying neuropathological lesions, such as cerebrospinal fluid biomarkers for $\mathrm{AD}$ or amyloid PET [53, 54], than from ever-increasing sophistication at the clinicalphenotypical level.

\section{Abbreviations \\ AD: Alzheimer's disease; BA: Brodmann area; CBD: corticobasal degeneration; FTLD: frontotemporal lobar degeneration; LV: logopenic variant; \\ NFV: nonfluent/agrammatic variant; PET: positron emission tomography; \\ PPA: primary progressive aphasia; PSP: progressive supranuclear palsy; \\ SV: semantic variant; TDP: Tar DNA binding protein 43.}

\section{Competing interests}

Rik Vandenberghe has received research grants from Research Foundation Flanders (FWO) and KU Leuven, has had a clinical trial agreement for phase 1 and 2 study between University Hospitals Leuven and GEHC, has received non-financial support from GEHC (provision of ${ }^{18} \mathrm{~F}$ - flutemetamol for conduct of investigator-driven trial free of cost), has a clinical trial agreement (local principal investigator) between University Hospitals Leuven and Merck, Forum, Roche, has a consultancy agreement between the KU Leuven R\&D and EliLilly. No non-financial conflicts of interest exist.

\section{Authors' contributions}

RV reviewed the literature and wrote the article.

\section{Acknowledgements}

We thank Rose Bruffaerts and Jolien Schaeverbeke for their helpful suggestions. Supported by Inter-University Attraction Pole P6/29 (RV) and P7/ 11 (RV), KU Leuven OT/12/097 (RV) and Programme Financing (RV), Fund for Scientific Research - Flanders (FWO) G.0660.09 N (RV), Stichting Alzheimer

Onderzoek grant number 15005. RV is senior clinical investigator of the FWO.

\section{Author details}

'Department of Neurosciences, Laboratory for Cognitive Neurology, KU Leuven, Leuven, Belgium. ${ }^{2}$ Neurology Department, University Hospitals Leuven, Leuven, Belgium. ${ }^{3}$ Alzheimer Research Centre KU Leuven, Leuven research Institute for Neuroscience \& Disease, University of Leuven, Leuven, Belgium.

Published online: 21 April 2016

\section{References}

1. Gorno-Tempini ML, Hillis AE, Weintraub S, Kertesz A, Mendez M, Cappa SF, et al. Classification of primary progressive aphasia and its variants. Neurology. 2011;76:1006-14.

2. Gorno-Tempini ML, Dronkers NF, Rankin KP, Ogar JM, Phengrasamy L, Rosen $\mathrm{HJ}$, et al. Cognition and anatomy in three variants of primary progressive aphasia. Ann Neurol. 2004;55:335-46.

3. Gorno-Tempini ML, Brambati SM, Ginex V, Gonar J, Dronkers NF, Marcone A, et al. The logopenic/phonological variant of primary progressive aphasia. Neurology. 2008;71:1227-34.

4. Grossman M. The non-fluent/agrammatic variant of primary progressive aphasia. Lancet Neurol. 2012;11:545-55.

5. Hodges JR, Patterson K. Semantic dementia: a unique clinicopathological syndrome. Lancet Neurol. 2007;6:1004-14.

6. Mesulam MM, Wieneke C, Thompson C, Rogalski E, Weintraub S. Quantitative classification of primary progressive aphasia at early and mild impairment stages. Brain. 2012;135:1537-53.

7. Foster NL, Chase TN, Fedio P, Patronas NJ, Brooks RA, Di Chiro G. Alzheimer's disease: focal cortical changes shown by positron emission tomography. Neurology. 1983;33:961-5. 
8. Haxby JV, Duara R, Grady CL, Cutler NR, Rapoport SI. Relations between neuropsychological and cerebral metabolic asymmetries in early Alzheimer's disease. J Cereb Blood Flow Metab. 1985;5:193-200.

9. Mesulam MM, Weintraub S, Rogalski EJ, Wieneke C, Geula C, Bigio EH. Asymmetry and heterogeneity of Alzheimer's and frontotemporal pathology in primary progressive aphasia. Brain. 2014;137:1176-92.

10. Gefen T, Gasho K, Rademaker A, Lalehzari M, Weintraub S, Rogalski E, et al. Clinically concordant variations of Alzheimer pathology in aphasic versus amnestic dementia. Brain. 2012;135:1554-65.

11. Ash S, Evans E, O'Shea J, Powers J, Boller A, Weinberg D, et al. Differentiating primary progressive aphasias in a brief sample of connected speech. Neurology. 2013;81:329-36.

12. Teichmann M, Kas A, Boutet C, Ferrieux S, Nogues M, Samri D, et al. Deciphering logopenic primary progressive aphasia: a clinical, imaging and biomarker investigation. Brain. 2013:136:3474-88.

13. Leyton CE, Ballard KJ, Piguet O, Hodges JR. Phonologic errors as a clinical marker of the logopenic variant of PPA. Neurology. 2014;82:1620-7.

14. Rohrer JD, Caso F, Mahoney C, Henry M, Rosen HJ, Rabinovici G, et al. Patterns of longitudinal brain atrophy in the logopenic variant of primary progressive aphasia. Brain Lang. 2013;127:121-6.

15. Foxe DG, Irish M, Hodges JR, Piquet O. Verbal and visuospatial span in logopenic progressive aphasia and Alzheimer's disease. J Int Neuropsychol Soc. 2013;19:247-53.

16. Leyton CE, Hodges JR. Towards a clearer definition of logopenic progressive aphasia. Curr Neurol Neurosci Rep. 2013;13:396.

17. Savage S, Hsieh S, Leslie F, Foxe D, Piguet O, Hodges JR. Distinguishing subtypes in primary progressive aphasia: application of the Sydney language battery. Dement Geriatr Cogn Disord. 2013;35:208-18.

18. Rohrer JD, Rossor MN, Warren JD. Alzheimer's pathology in primary progressive aphasia. Neurobiol Aging. 2012;33:744-52.

19. Leyton CE, Britton AK, Hodges JR, Halliday GM, Krill JJ. Distinctive pathological mechanisms involved in primary progressive aphasias. Neurobiol Aging. 2016;38:82-92.

20. Ueno T, Saito S, Rogers TT, Lambon Ralph MA. Lichtheim 2: synthesizing aphasia and the neural basis of language in a neurocomputational model of the dual dorsal-ventral language pathways. Neuron. 2011;72:385-96.

21. Nelissen N, Pazzaglia M, Vandenbulcke M, Sunaert S, Fannes K, Dupont P, et al. Gesture discrimination in primary progressive aphasia: the intersection between gesture and language processing pathways. J Neurosci. 2010;30: 6334-41.

22. Galantucci S, Tartaglia MC, Wilson SM, Henry ML, Filippi M, Agosta F, et al. White matter damage in primary progressive aphasias: a diffusion tensor tractography study. Brain. 2011;134:3011-29.

23. Adamczuk K, De Weer AS, Nelissen N, Dupont P, Sunaert S, Bettens K, et al. Functional changes in the language network in response to increased amyloid $\beta$ deposition in cognitively intact older adults. Cereb Cortex. 2016;26(1):358-73.

24. Nelissen $N$, Vandenbulcke $M$, Fannes $K$, Verbruggen $A$, Peeters $R$, Dupont $P$, et al. Abeta amyloid deposition in the language system and how the brain responds. Brain. 2007;130:2055-69.

25. Bonner MF, Grossman M. Gray matter density of auditory association cortex relates to knowledge of sound concepts in primary progressive aphasia. J Neurosci. 2012;32:7986-91.

26. Grossman M. Primary progressive aphasia: clinicopathological correlations. Nat Rev Neurol. 2010:6:88-97.

27. Harris JM, Gall C, Thompson JC, Richardson AMT, Neary D, du Plessis D, et al. Classification and pathology of primary progressive aphasia. Neurology. 2013;81:1832-9.

28. Leyton CE, Villemagne VL, Savage S, Pike KE, Ballard KJ, Piguet O, et al. Subtypes of progressive aphasia: application of the International Consensus Criteria and validation using $\beta$-amyloid imaging. Brain. 2011;134:3030-43.

29. Hoffman P, Jones RW, Lambon-Ralph MA. The degraded concept representation system in semantic dementia: damage to pan-modal hub, then visual spoke. Brain. 2012:135:3770-80

30. Mesulam MM, Wieneke C, Hurley R, Rademaker A, Thompson CK, Weintraub $\mathrm{S}$, et al. Words and objects at the tip of the left temporal lobe in primary progressive aphasia. Brain. 2013;136:601-18.

31. Henry ML, Beeson PM, Alexander GE, Rapcsak SZ. Written language impairments in primary progressive aphasia: a reflection of damage to central semantic and phonological processes. J Cogn Neurosci. 2012;24:261-75
32. Kumfor F, Landin-Romero R, Devenney E, Hutchings R, Grasso R, Hodges JR, et al. On the right side? A longitudinal study of left- versus right-lateralized semantic dementia. Brain. 2016. http://dx.doi.org/10.1093/brain/awv387.

33. Davies RR, Graham KS, Xuereb JH, Williams GB, Hodges JR. The human perirhinal cortex and semantic memory. Eur J Neurosci. 2004;20:2441-6.

34. Mion M, Patterson K, Acosta-Cabronero J, Pengas G, Izquierdo-Garcia D, Hong YT, et al. What the left and right anterior fusiform gyri tell us about semantic memory. Brain. 2010;133:3256-68.

35. La Joie R, Landeau B, Perrotin A, Bejanin A, Egret S, P'elerin A, et al. Intrinsic connectivity identifies the hippocampus as a main crossroad between Alzheimer's and semantic dementia-targeted networks. Neuron. 2014;81:1417-28.

36. Bruffaerts R, Dupont P, Peeters R, De Deyne S, Storms G, Vandenberghe R. Similarity of fMRI activity patterns in left perirhinal cortex reflects semantic similarity between words. J Neurosci. 2013:33:18597-607.

37. Agosta F, Henry RG, Migliaccio R, Neuhaus J, Miller BL, Dronkers NF, et al. Language networks in semantic dementia. Brain. 2010;133:286-99.

38. Guo CC, Gorno-Tempini ML, Gesierich B, Henry M, Trujillo A, Shany-Ur T, et al. Anterior temporal lobe degeneration produces widespread networkdriven dysfunction. Brain. 2013;136:2979-91.

39. Josephs KA, Hodges JR, Snowden JS, Mackenzie IR, Neumann M, Mann DM, et al. Neuropathological background of phenotypical variability in frontotemporal dementia. Acta Neuropathol. 2011;122:137-53.

40. Davies RR, Hodges JR, Kril JJ, Patterson K, Halliday GM, Xuereb JH. The pathological basis of semantic dementia. Brain. 2005;128:1984-95.

41. Josephs KA, Duffy JR, Strand EA, Machulda MM, Senjem ML, Master AV, et al. Characterizing a neurodegenerative syndrome: primary progressive apraxia of speech. Brain. 2012;135:1522-36.

42. Josephs KA, Duffy JR, Strand EA, Machulda MM, Senjem ML, Lowe VJ, et al. Syndromes dominated by apraxia of speech show distinct characteristics from agrammatic PPA. Neurology. 2013:81:337-45.

43. Ballard KI, Savage S, Leyton CE, Vogel AP, Hornberger M, Hodges JR. Logopenic and nonfluent variants of primary progressive aphasia are differentiated by acoustic measures of speech production. PLoS One. 2014;9, e89864.

44. Josephs KA, Duffy JR, Strand EA, Machulda MM, Senjem ML, Gunter JL, et al. The evolution of primary progressive apraxia of speech. Brain. 2014;137:2783-95

45. Whitwell JL, Duffy JR, Strand EA, Xia R, Mandrekar J, Machulda MM, et al. Distinct regional anatomic and functional correlates of neurodegenerative apraxia of speech and aphasia: an MRI and FDG-PET study. Brain Lang. 2013:125:245-52.

46. Catani M, Mesulam MM, Jakobsen E, Malik F, Martersteck A, Wieneke C, et al. A novel frontal pathway underlies verbal fluency in primary progressive aphasia. Brain. 2013;136:2619-28.

47. Mandelli ML, Caverzasi E, Binney RJ, Henry ML, Lobach I, Block N, et al. Frontal white matter tracts sustaining speech production in primary progressive aphasia. J Neurosci. 2014:34:9754-67.

48. Wilson SM, Galantucci S, Tartaglia MC, Rising K, Patterson DK, Henry ML, et al. Syntactic processing depends on dorsal language tracts. Neuron. 2011;72:397-403.

49. Wicklund MR, Duffy JR, Strand EA, Machulda MM, Whitwell JL, Josephs KA. Quantitative application of the primary progressive aphasia consensus criteria. Neurology. 2014:82:1119-26.

50. Sajjadi SA, Patterson K, Arnold RJ, Watson PC, Nestor PJ. Primary progressive aphasia: a tale of two syndromes and the rest. Neurology. 2012;78:1670-7.

51. Sajjadi SA, Patterson K, Nestor PJ. Logopenic, mixed, or Alzheimer-related aphasia? Neurology. 2014;82:1127-31.

52. Mesulam MM, Weintraub S. Is it time to revisit the classification guidelines for primary progressive aphasia? Neurology. 2014;82:1108-9.

53. Vandenberghe R, Adamczuk K, Dupont P, Laere KV, Ch'etelat G. Amyloid PET in clinical practice: Its place in the multidimensional space of Alzheimer's disease. Neuroimage Clin. 2013;2:497-511.

54. Vandenberghe R. The relationship between amyloid deposition, neurodegeneration, and cognitive decline in dementia. Curr Neurol Neurosci Rep. 2014:14:498. 